\title{
LAS TRANSFORMACIONES SOCIODEMOGRÁFICAS $Y$ LA VULNERABILIDAD EN LOS PROCESOS DE LARGA DURACIÓN.
}

\section{Dra. Ana María H. Foschiatti}

- Investigadora del Consejo Nacional de Investigaciones Científicas y Técnicas (CONICET)

- Directora del Instituto de Geografía, Facultad de Humanidades - Universidad Nacional del Nordeste (UNNE) Argentina.

- Profesora Titular de la Cátedra Geografía de la Población, Facultad de Humanidades, Universidad Nacional del Nordeste (UNNE) Argentina

- Avda. Las Heras 727 - 3500 - Resistencia - Chaco - Argentina

-Tel-Fax: 54 - 3722 - 446958 / 422257 / 432283

-E-mail: amfoschiatti@hum.unne.edu.ar 


\title{
LAS TRANSFORMACIONES SOCIODEMOGRÁFICAS Y LA VULNERABILIDAD EN LOS PROCESOS DE LARGA DURACIÓN.
}

\begin{abstract}
Resumen
En los albores del siglo XXI se plantea reiteradamente el tema de la vulnerabilidad sociodemográfica en el estudio de los distintos procesos que involucran a las sociedades humanas, como sí también en los debates sobre políticas públicas dedicadas a reducir la pobreza. Su difusión obedeció a factores y fenómenos muy variados, entre los que figuran la creciente inestabilidad económica, la calidad de vida de las poblaciones, los signos de fragmentación social, la indefensión ante riesgos de diversa naturaleza y la dificultad del acceso de los activos a la movilidad social

Esto nos induce a la consideración de esas características en los procesos de cambio demográfico que operan en períodos de tiempo relativamente extensos y tienen la peculiaridad de iniciarse en algunos espacios geográficos y expandirse luego a otros. Los cuatro procesos de duración prolongada que se analizan en este artículo y se los vincula con la vulnerabilidad sociodemográfica son: la transición demográfica, la transición urbana y de la movilidad, la segunda transición demográfica y la transición epidemiológica que estructuran las condiciones demográficas presentes y futuras.
\end{abstract}

\section{THE TRANSFORMATIONS SOCIODEMOGRÁFICAS AND THE VULNERABILITY IN THE PROCESSES OF LONG DURATION.}

\begin{abstract}
In the dawn del century XXI repeatedly considers the subject of the sociodemográfica vulnerability in the study of the different processes that involve the human societies, as yes also in the debates on public policies dedicated to reduce the poverty. Its diffusion obeyed to factors and phenomena very varied, between which they appear the increasing economic instability, the quality of life of the populations, the signs of social fragmentation, the defenselessness before risks of diverse nature and the difficulty of the access of the assets to social mobility

This induces us to the consideration of those characteristics in the processes of demographic change that operate in relatively extensive periods of time and have the peculiarity to begin in some geographic spaces and to expand soon to others. The four processes of prolonged duration that are analyzed in this article and tie them with the sociodemográfica vulnerability are: the demographic transition, the urban transition and of mobility, the second demographic transition and the transition epidemiologist whom the present and future demographic conditions structure.
\end{abstract}




\section{LAS TRANSFORMACIONES SOCIODEMOGRÁFICAS Y LA VULNERABILIDAD EN LOS PROCESOS DE LARGA DURACIÓN.}

\section{Introducción}

La Geografía de la población se distingue por su capacidad para anticipar sucesos demográficos, incluso los que llevan implícito riesgos sociales. Ello ocurre principalmente porque los procesos de cambio demográfico operan en relativamente extensos períodos de tiempo y tienen la particularidad de comenzar en algunas áreas y propagarse a otras. Cuatro procesos de larga duración configuran de manera decisiva las condiciones demográficas pasadas, presentes y futuras. Su vinculación con la vulnerabilidad sociodemográfica se da por el hecho que de ellos se desprenden los rasgos actuales y los que probablemente influirán en el futuro para generar situaciones que implicarán algún riesgo. En la vulnerabilidad sociodemográfica se conjugan sucesos riesgosos, la incapacidad para responder a los mismos y la inhabilidad para adaptarse a la nueva situación generada a partir de ellos. Si bien los tres enunciados son importantes, el riesgo sólo puede explicarse mediante las variables de población, particularmente con los procesos de larga duración; los otros son circunstanciales y dependen de las condiciones sociales del grupo. Esos procesos de cambios son: la transición demográfica, la transición urbana y de la movilidad, la segunda transición demográfica y la transición epidemiológica.

La transición demográfica se caracteriza por el descenso sostenido de la natalidad y la mortalidad desde niveles altos a bajos, lo que se traduce a largo plazo en un cambio poblacional que se expresa, al finalizar el proceso, en un lento crecimiento de la población y en un cambio en la estructura etaria (envejecimiento demográfico). La transición urbana y de la movilidad consiste en el incremento acelerado de la proporción de la población urbana a lo que se agrega la importancia de los movimientos migratorios dentro y fuera de las ciudades y los desplazamientos largos (internos e internacionales). Por otra parte, la segunda transición demográfica puede sintetizarse en los siguientes hechos: cambios en la estructura familiar, postergación de las iniciaciones nupciales y reproductivas (a veces definitivas) y a la transformación de la institución matrimonial (más informal y frágil). Mientras que la transición epidemiológica se da en forma paralela a la transición demográfica e implica el paso de enfermedades y causas de muerte infecciosas y transmisibles (parasitarias) hacia enfermedades degenerativas y no transmisibles (tumores, violencia); transformaciones que ocurren debido a cambios en el comportamiento en el plano de la salud, la nutrición, avances en la medicina y cambios socioambientales. El envejecimiento de la población también desempeña un rol importante en el cambio de perfil epidemiológico, porque son los adultos mayores los más vulnerables a las patologías crónicas y a las enfermedades degenerativas, es así que ante una mayor esperanza de vida y una reducción de las enfermedades transmisibles y neonatales, la estructura epidemiológica va cambiando paulatinamente.

\section{Los procesos de cambio demográfico}

\section{a. La transición demográfica}

Las transformaciones demográficas observadas en los países desarrollados estuvieron vinculadas a las transformaciones económicas y al desarrollo de los cambios del nivel de vida de la población. Ese proceso conocido como transición demográfica consistió en el paso de altas tasas de natalidad y mortalidad a niveles muy bajos de ambos. (Chackiel, 2004)

Generalmente, para describir las características básicas de la dinámica de la población, se utiliza ese concepto de transición demográfica. Dicho proceso, que nace de lo ocurrido en los cambios demográficos de los países europeos (como resultado de las transformaciones económicas y sociales después de la Revolución industrial) permite ubicar a los países en cuatro etapas de la transición. Esas etapas se diferencian por los valores de las tasas de natalidad y mortalidad mostradas a través de los años con ciertas características de índole social y espacial. (Rivadeneira, 2000, 22)

América Latina experimentó en mayor o menor medida ese paso -aunque le tomó menos tiempo- observándose diferencias muy notables en los distintos países (países en proceso incipiente o en estado avanzado). El mismo está relacionado con el crecimiento económico, las transformaciones sociales y la incorporación de tecnología ya disponible; acontecimiento que a los países más avanzados les llevó mucho más tiempo generarlos. 
Las etapas de la transición demográfica se vinculan con los diferentes ritmos del crecimiento natural (que determinan su velocidad), con las distintas estructuras de edades (que inciden en la demanda de viviendas y en la cantidad de hogares), con los niveles de distribución urbano-rural de la población (que repercuten en la provisión de servicios y tenencia de la tierra).

El descenso de la mortalidad general e infantil se vincula con la mejoría del saneamiento de las viviendas. La disminución de la fecundidad se debe a las modificaciones del modelo de familia, a los cambios de la estructura de edades y a las nuevas pautas de comportamiento reproductivo. Además la transición demográfica está asociada a la transición urbana (aumento de la urbanización y cambios en los patrones de desplazamiento territorial) que en definitiva son indicadores de la magnitud de los requerimientos socioeconómicos.

Existen numerosos estudios sobre la transición demográfica que explican las características particulares del proceso que comprende la modificación de cuatro riesgos: la mortalidad, la fecundidad, la estructura etaria y la dependencia. El descenso de los dos primeros compromete directamente a los individuos y es indicativo de mejores condiciones de salud y de capital humano, mientras que los otros dos se refieren a la sociedad en su conjunto. En la estructura por edades de la población se observa un marcado rejuvenecimiento, seguido de un abultamiento de las edades intermedias, para luego finalizar con un acrecentamiento de la población mayor. En relación con este comportamiento, el proceso de transición en las distintas regiones, difiere en el momento de inicio, en la velocidad en que se producen los cambios, en los valores de los índices y en la incidencia de las variables conexas. (CEPAL, 2002)

La primera etapa, de transición incipiente, se caracteriza por condiciones de pobreza con población predominantemente rural, deficiente acceso a los servicios sociales; con tasas de crecimiento natural del $2.5 \%$, índices de natalidad y mortalidad elevados y sostenidos y una alta proporción de niños y jóvenes. (Rivadeneira, 2000)

En una segunda etapa, de transición moderada, si bien diminuye la mortalidad con un rejuvenecimiento de la población y un aumento de los índices de dependencia, no se logró la misma conducta en la mayoría de la población rural y en aquellos segmentos sociales en situación de pobreza. Como resultado de una mortalidad en descenso y una natalidad elevada y sostenida se registran tasas de crecimiento natural cercanas al 3\% anual. Dado el importante volumen de población infantil y juvenil con respecto a la población total, es probable que se siga manifestando una natalidad elevada.

En la etapa de plena transición se registra un predominio urbano, una natalidad en descenso y una mortalidad que disminuyó considerablemente debido a su joven estructura etaria y a las campañas de salud realizadas. El crecimiento medio natural de estos países es cercano al $2 \%$ anual.

Por último se proyecta la etapa de transición avanzada con un alto grado de urbanización, con tasas de crecimiento natural anual del orden del $1 \%$, natalidad baja y mortalidad moderada o baja. Algunas áreas se caracterizan por una importante proporción de población anciana, como producto del descenso en los niveles de fecundidad.

En América Latina, comenzó a descender la mortalidad con mayor intensidad desde 1930, y se afianza con posterioridad a la Segunda Guerra Mundial. La región alcanzó una $e_{\circ}$ promedio de 52 años y una tasa de mortalidad infantil de 127 por mil a mediados del siglo XX. En las décadas siguientes se logró un descenso mayor, superando una $e_{o}$ de 60 años (1970) y de 70 años (2000) con una tasa de mortalidad infantil de 36 por mil. (Chackiel - Schkolnik, 2003)

El descenso de la fecundidad fue posterior. A mediados del siglo fue de 6 hijos por mujer con tendencia ascendente por efectos del descenso de la mortalidad que exponía a la mujer a quedar embarazada a edades más avanzadas, además de la mejora en las condiciones de salud. Recién a partir de 1960 se produce un cambio, llegando en la actualidad a 2,7 hijos por mujer. Ese cambio es la respuesta a la revolución anticonceptiva que dio comienzo en Europa con resultado de bajas, incluso por debajo de los niveles de reemplazo.

El avance de la transición demográfica reduce riesgos cruciales, pues amplia el horizonte vital de las personas y su grado de control sobre la reproducción; además, a mediano plazo genera el denominado "bono demográfico" u "oportunidad demográfica" que implica el cambio en los índices de dependencia hacia una menor proporción entre la población activa y la inactiva. De esa manera se 
espera que haya una reducción de las personas en edad no laboral, lo que implicaría mayor fuerza de trabajo, generación de ahorros, mejoras en la inversión y mayor ingreso per capita. (Busso, 2002)

La rápida transición demográfica que se experimentó en los países en vías de desarrollo, modifica el perfil que adquieren los riesgos. Desde mediados del siglo XX se produjo el descenso de la fecundidad y de la mortalidad, se debilitó el movimiento natural de la población y estrechó la base de la pirámide, marcando una disminución del número de niños en los hogares, es decir aumentó el control sobre la salud y la reproducción de las personas, quedando atrás las amenazas de un elevado crecimiento y de una población juvenil altamente vulnerable. Este proceso se extiende en todas las clases sociales y en todas las regiones, aunque se observa un rezago del proceso en algunos países con menor capacidad de respuesta para su materialización o con poca capacidad para adaptarse a los riesgos sociodemográficos, exhibiendo sesgos de género y generación. Por lo tanto el rezago transicional puede aumentar la vulnerabilidad de los hogares y las personas, de allí que en su avance se pueden reducir los riesgos. No obstante, esta conclusión puede ser matizada por tres razones: a) el avance de la transición no atenúa todos los riesgos (maternidad adolescente) y emergen otros (envejecimiento), b) existen riesgos sociodemográficos que dependen de la transición urbana y de la segunda transición, c) el desarrollo de la misma no asegura la disminución de la vulnerabilidad social, pues existen numerosos riesgos que escapan de la esfera demográfica, como así tampoco garantiza el logro de aspiraciones sociales y económicas de las personas.

El tema de la maternidad adolescente tiene consecuencias adversas para las involucradas, pues ellas se ven en la necesidad de asumir roles e improvisar decisiones para los cuales no están preparadas, todo lo cual resulta desventajoso para la estabilidad familiar y la crianza de los hijos. Además la sociedad sufre la salida del sistema educativo de los recursos humanos que aún están en pleno proceso de formación. Esa reproducción temprana es un riesgo relevante en numerosas áreas y obedece a patrones de conducta y normas socioculturales que implican distintas condiciones de vulnerabilidad y es un componente demográfico de la pobreza que implica un cuadro de vulnerabilidad, pues afecta a los que poseen menor capacidad de respuesta y defensa.

La transición demográfica genera nuevos riesgos que modifican la estructura por edad de la población: la oscilación de las cohortes y el proceso de envejecimiento. El primero se origina en las variaciones del número de nacimientos que cambian el tamaño de los grupos de edades a medida que las cohortes avanzan en su ciclo vital. Es así que el descenso acelerado de la fecundidad causa grandes fluctuaciones en el tamaño de las mismas, especialmente cuando el descenso es irregular. Los riesgos que ello trae aparejado afectan a todas las comunidades que están en proceso de transición demográfica. El segundo riesgo es el envejecimiento cuyas consecuencias adversas se hacen sentir sobre las variables económicas, las finanzas, el perfil epidemiológico y la estructura social. Este último proceso está en marcha en muchas regiones y muestra el aumento de la proporción y el volumen del grupo de población de 60 años y más. Aunque el envejecimiento cronológico es un riesgo inevitable, el demográfico puede manejarse mediante el control de los factores determinantes, como la fecundidad y las migraciones, sin embargo ello depende de la capacidad de respuesta de las comunidades afectadas. Por lo general las áreas o países más envejecidos tienen niveles más altos de desarrollo aunque lejos del que presenta las áreas o países adelantados ya que no hay antecedentes de envejecimiento demográfico en un contexto precario.

En otro orden de cosas, el envejecimiento puede considerarse como un riesgo con perfiles específicos en los hogares y en las personas vinculada con la pérdida de recursos, con el deterioro físico y mental en las etapas posteriores de la vida. Es decir que, a pesar de que el aumento de la expectativa de vida es una señal alentadora para la población, tanto por su significado intrínseco personal como socioeconómico, no siempre va acompañado de una mejor calidad de vida, pues es cada vez mayor la cantidad de hogares y personas que envejece en un contexto precario.

Un riesgo sociodemográfico que se debilita con el avance de la transición demográfica es, precisamente, la mortalidad. Ello significa que se pueden evitar muertes por causas previsibles y además, postergar la vida de las personas hasta el límite máximo que permite la capacidad humana y médica. En ese sentido la transición demográfica está estrechamente vinculada con la transición epidemiológica, aunque su avance no es sinónimo de desaparición de riesgos de morbimortalidad evitable, como fácilmente se observa en numerosas áreas y regiones con profundas desigualdades socioeconómicas. La vulnerabilidad no solo se manifiesta en la rápida diversificación de los grupos expuestos, sino en la diversidad socioeconómica que determina la posibilidad de prevenir las enfermedades y en la capacidad de respuesta y adaptación a las mismas. 
La mortalidad y los diversos grados de impedimentos que ocasionan los accidentes y los modos de violencia no cambian con el avance de la transición demográfica, pues no existe una dependencia con ella. Muchos acontecimientos pueden prevenirse mediante las modificaciones a largo plazo de la conducta y de los factores culturales de los hogares y las personas. El siguiente cuadro sintetiza los riesgos propios de las diferentes etapas de la transición demográfica y algunas condiciones necesarias para reducir la vulnerabilidad demográfica y social:

\section{Cuadro No1: Transición demográfica: Características generadoras de riesgos y medidas necesarias para reducir la vulnerabilidad (Busso, 2002)}

\begin{tabular}{|c|c|c|}
\hline $\begin{array}{c}\text { Etapas de } \\
\text { la } \\
\text { transición }\end{array}$ & Cualidades generadoras de riesgos & $\begin{array}{c}\text { Medidas necesarias para reducir la } \\
\text { vulnerabilidad }\end{array}$ \\
\hline $\begin{array}{l}\text { Transición } \\
\text { incipiente }\end{array}$ & $\begin{array}{ll}\text { - } & \text { Baja urbanización } \\
\text { - } & \text { Elevada proporción de población joven } \\
\text { - } & \text { Predominio de enfermedades transmisibles, } \\
\text { infecciosas y parasitarias } \\
\text { - } & \text { Altas tasas de dependencia demográfica } \\
\text { Bajos niveles de instrucción y alta deserción } \\
\text { - } \quad \text { Altos niveles de pobreza urbana y rural } \\
\text { - } \quad \text { Proliferación de áreas marginales habitadas } \\
\quad \text { por inmigrantes rurales }\end{array}$ & $\begin{array}{l}\text { - } \quad \text { Proporcionar infraestructura rural } \\
\text { - } \quad \text { Acuidado de la salud materno infantil } \\
\text { - } \quad \text { básica y secundaria } \\
\text { - } \quad \text { Disponibilidad de viviendas y servicios } \\
\text { - } \quad \text { Capbanos } \\
\text { - }\end{array}$ \\
\hline $\begin{array}{l}\text { Transición } \\
\text { moderada }\end{array}$ & $\begin{array}{l}\text { - } \quad \text { Urbanización baja y moderada } \\
\text { Proliferación de suburbios marginales } \\
\text { habitados por inmigrantes rurales } \\
\text { Rejuvenecimiento de la población } \\
\text { - } \quad \text { Embarazo adolescente } \\
\text { Predominio de enfermedades transmisibles, } \\
\text { infecciosas y parasitarias }\end{array}$ & $\begin{array}{ll}\text { - } & \text { Disponibilidad de viviendas } \\
\text { - } & \text { Infraestructura urbana y rural } \\
\text { - } & \text { Equipamiento sanitario materno infantil } \\
\text { - } & \text { Atención a la salud reproductiva } \\
\text { - } & \text { Cobertura y calidad de la educación } \\
\text { - } & \text { Marginalidad juvenil } \\
\text { - } & \text { Aumento de la capacidad del empleo }\end{array}$ \\
\hline $\begin{array}{l}\text { Plena } \\
\text { transición }\end{array}$ & $\begin{array}{l}\text { - } \quad \text { Aumento de población joven y adulta } \\
\text { - } \quad \text { Embanización moderada y alta } \\
\text { - } \\
\text { Enfermedades infecciosas, parasitarias y } \\
\text { crónicas } \\
\text { Exclusión y marginación de amplios } \\
\text { espacios urbanos carentes de infraestructura } \\
\text { y servicios }\end{array}$ & $\begin{array}{l}\text { - } \quad \text { Educación secundaria y superior } \\
\text { - } \quad \text { Disponibilidad de empleo } \\
\text { - } \quad \text { Salud de adultos } \\
\text { - } \quad \text { Cobertura y atención a la salud } \\
\text { reproductiva Acceso a la vivienda digna } \\
\text { - } \quad \text { Disponibilidad de infraestructura urbana } \\
\text { - Salud materno infantil e implementación } \\
\text { de sistemas de alta complejidad } \\
\text { - Previsión para la vejez }\end{array}$ \\
\hline $\begin{array}{l}\text { Transición } \\
\text { avanzada }\end{array}$ & $\begin{array}{l}\text { - } \quad \text { Altos valores de urbanización } \\
\text { - } \quad \text { Prejecimiento demográfico } \\
\text { degenerativas; aumento de incidencia de } \\
\text { causas de muerte por factores externos } \\
\text { (accidentes, homicidios, suicidios, etc.) } \\
\text { - Cambio en los patrones familiares y } \\
\text { reproductivos } \\
\text { - } \quad \text { Aumesprotección de ancianos } \\
\text { sistema de pensiones }\end{array}$ & $\begin{array}{ll}\text { - } & \text { Acceso a la vivienda } \\
\text { - } & \text { Infraestructura urbana } \\
\text { - } & \text { Aumento del empleo } \\
\text { - } & \text { Educación superior } \\
\text { - } & \text { Implementación de atención de alta } \\
& \text { complejidad } \\
\text { - } & \text { Implementación de pensiones } \\
& \text { Recreación y contención para adultos } \\
\text { mayores } \\
\text { - Cobertura y financiamiento de sistemas } \\
\text { de pensiones }\end{array}$ \\
\hline
\end{tabular}




\section{b. La transición urbana y de la movilidad}

La transición urbana se explica por el aumento sostenido de la proporción de población urbana y el estancamiento demográfico de las áreas rurales, que es acompañado por un cambio de las conductas de movilidad (traslado entre las ciudades y dentro de ellas). Por otra parte, la fecundidad, la mortalidad, las migraciones y las complejas interacciones entre población, medio ambiente y organización económica definen los patrones de distribución de la población y la ocupación del territorio. La migración forma parte de la historia de esa ocupación y del comportamiento de las personas cuando no encuentran oportunidades para satisfacer sus necesidades.

La urbanización acelerada y las elevadas proporciones de población viviendo en las ciudades son características inherentes al siglo XX. Por su importancia demográfica, sus causas y sus consecuencias, dicho fenómeno puede ser considerado como un proceso de redistribución espacial. El paso de una situación con predominio rural hacia 1950 a otra con mayoría urbana es una de las más destacadas expresiones de los profundos cambios provocados por la redistribución espacial. A mediados del Siglo XX, en América Latina, la población urbana alcanzaba al 40\%, mientras que en la segunda mitad del siglo esta situación consolida a la región como la más urbanizada del mundo con valores que superan el $75 \%$ a pesar de la desaceleración del ritmo de crecimiento de dicha población. (Rodríguez Vignoli, 2002)

Los riesgos relacionados con las distintas etapas de la transición urbana y de la movilidad se sintetizan en las imágenes de "colapso urbano" y "desierto rural". La migración rural-urbana encierra una serie de situaciones desfavorables tanto para las áreas urbanas como para los propios migrantes que sufren situaciones de incertidumbre y de fragilidad en su inserción al medio. Por otra parte el despoblamiento del campo es otro riesgo que se debe enfrentar en las etapas iniciales de la transición urbana, aunque la población rural continúa expandiéndose como resultado del crecimiento natural más elevado. En etapas más avanzadas de esa transición se observa que los riesgos y problemas de la sociedad se "urbanizan" a través de la aparición de otros fenómenos como la criminalidad, la contaminación, la violencia, riesgos contingentes que pueden desaparecer. Al mismo tiempo, y en etapas posteriores se pueden promover aspectos positivos como el "bono demográfico" a causa de la selectividad migratoria, aunque la urbanización modifica el perfil de los riesgos sociales, generándose riesgos sociodemográficos como la segregación residencial y el crecimiento desordenado. (Busso, 2002)

En síntesis, independientemente de la etapa de transición urbana que se trate, la localización de la población conduce a la existencia de dos riesgos fundamentales: la dispersión de la población rural con la proliferación de ciudades de pequeño tamaño y la instalación de las personas en áreas precarias o expuestas a problemas ambientales.

Algunos de los riesgos urbanos se vinculan con la organización de los sistemas primados de ciudades o ciudades grandes con predominio de riesgos sociodemográficos relacionados con la localización y la movilidad dentro de las ciudades.

En numerosos sistemas urbanos de América Latina sobresalen las ciudades grandes y su presencia tiene raíces históricas. La vocación urbana de los conquistadores provocó la fundación de ciudades con funciones de defensa y poder político - económico. En el año 2000 el número de ciudades de más de un millón de habitantes alcanzaba a las 49; de ellas, 7 urbes superaban los 5 millones y 4 los 10 millones. Asimismo la evolución de la población de las ciudades fue acelerada y no cabe duda que ellas jugaron un papel importante en el proceso de redistribución de la población a través de la transferencia de personas del campo a la ciudad por procesos migratorios. La elevada concentración de población en las ciudades principales es un atributo de los sistemas urbanos de los países subdesarrollados (primacía urbana). (Rodríguez Vignoli, 2002)

El proceso de urbanización en los países de Latinoamérica tiene las siguientes características hasta 1970: alto crecimiento demográfico debido a la alta fecundidad y a la importante migración campo-ciudad, la reclasificación de los espacios rurales y a la tendencia de la concentración de la población en las ciudades grandes. A partir de 1980 se observan cambios debido principalmente a la disminución de los niveles de fecundidad y a la reducción de la migración rural, que permitieron la desconcentración de las áreas metropolitanas y el incremento de las ciudades intermedias. (Pinto da Cunha, 2002) 
Estas últimas ciudades son uno de los componentes más destacados y dinámicos del sistema urbano (entre 50 mil y un millón de habitantes). La cantidad y heterogeneidad de las mismas dificultan los análisis detallados, a la vez que son afectadas muy rápidamente por sucesos puntuales como inundaciones, instalación o cierre de industrias, flujos migratorios, entre otros.

En otro orden de cosas el despoblamiento de las áreas centrales, la segregación residencial o la ocupación de tierras suburbanas expuestas a todo tipo de riesgos se relacionan con la conectividad y la precariedad de los servicios. No es un fenómeno nuevo que el ritmo de crecimiento de las periferias urbanas es mucho más intenso que la zona central; las fuerzas que conducen a esta situación están relacionadas con la saturación del espacio central, el elevado costo de los terrenos o la ocupación paulatina del centro por funciones productivas o comerciales. Pero esa expansión periférica difiere de las características de suburbanización de países desarrollados, mientras que en estos se reserva la zona central para la instalación de actividades no residenciales y grupos pobres o segregados y las áreas suburbanas para grupos acomodados, en los países latinoamericanos son las periferias ampliadas las que albergan a los más pobres o a los migrantes rurales, que son los responsables de la ampliación de los suburbios.

La expansión se debe además a la demanda habitacional insatisfecha (instalaciones espontáneas, invasiones) a la erradicación forzosa de asentamientos, a programas de vivienda construidas donde los terrenos son más baratos, que acumulan deficiencias y postergaciones (niveles de vida inferiores a los promedios, equipamiento escaso, deficitaria accesibilidad y precariedad de los transportes, vulnerabilidad ambiental, riesgos naturales, gastos en desplazamientos, etc.).

El asentamiento precario es una forma de hábitat generado por las dificultades para acceder a la propiedad de los terrenos urbanos (ocupación informal) en áreas con riesgo ambiental, en terrenos públicos que provocan la inseguridad de la tenencia, déficit de los servicios básicos, riesgo sanitario, entre otros. Una gran proporción de los hogares tiene la combinación de dos factores de vulnerabilidad: un bajo nivel del jefe del hogar y alta dependencia económica, que implica también riesgo para la salud. (Arriagada Luco, 2003)

El hacinamiento definido como el problema de sobrecarga del parque habitacional causado por el elevado número de habitantes en las viviendas respecto a la cantidad de habitaciones (tres o más personas por cuarto destinado a dormitorio) tiene los peores índices en los sectores con transición rezagada. Es destacable notar que el hacinamiento es un factor que interfiere en la adquisición de capital educativo de las personas y favorece al crecimiento de la pobreza. (Arriagada Luco, 2003).

A medida que avanzaron la transición urbana y la transición demográfica se transitó hacia una reducción del crecimiento de las ciudades más grandes, aunque estas siguen conteniendo a gran parte de la población total y urbana. Las mayores aglomeraciones de los países latinoamericanos muestran una expansión demográfica importante de las áreas periféricas, mientras las áreas centrales reducen su crecimiento. Ello no se debe a la reducción de la migración rural ni al descenso del crecimiento vegetativo, sino a los procesos de redistribución dentro de las ciudades de magnitud y complejidad crecientes. Actualmente y en las urbes de mayor tamaño, el proceso de segregación residencial hacia las áreas alejadas es un denominador común, desde finales del siglo XX.

El proceso de transición urbana conlleva un descenso sostenido de la fecundidad y la mortalidad, aunque en las áreas con una fase incipiente de ese proceso se complica el panorama pues se registran altos niveles de crecimiento demográfico y rural con un bajo desarrollo socioeconómico. Esto último, unido a los serios problemas de dispersión y aislamiento continúa en las fases más avanzadas de la transición.

A partir de las diferencias entre los hogares urbanos y rurales, pobres y no pobres, en el trabajo de Busso (2002, 53-58) se identifican cinco formas básicas de vulnerabilidad en una sociedad: Vulnerabilidad alta (VA), vulnerabilidad media-alta (VMA), vulnerabilidad media (VM), vulnerabilidad media-baja (VMB) y vulnerabilidad baja (VB). Las características básicas se sintetizan el siguiente cuadro: 
Cuadro No2: Vulnerabilidad de los hogares: características y políticas de mejoramiento

\begin{tabular}{|c|c|c|}
\hline $\begin{array}{l}\text { Vulnerabilidad } \\
\text { de la población }\end{array}$ & Rasgos preponderantes & Medidas de mejoramiento \\
\hline $\begin{array}{c}\text { Vulnerabilidad } \\
\text { alta }\end{array}$ & $\begin{array}{l}\text { - } \quad \text { Elevado crecimiento natural } \\
\text { - } \quad \text { Índice de masculinidad elevado } \\
\text { - } \quad \text { Mano de obra urbana de origen rural no } \\
\text { calificada } \\
\text { - } \quad \text { Predominio de actividades primarias } \\
\text { - } \quad \text { Existencia de dos o más NBI } \\
\text { - } \quad \text { Hacinamiento de los hogares } \\
\text { - } \quad \text { Pobreza rural } \\
\text { - } \quad \text { ulevados niveles de migración rural- } \\
\text { - } \quad \text { Elevada morndices de dependencia } \\
\text { - } \quad \text { mortalidad infantil, en la niñez y materna. } \\
\text { - } \quad \text { Baja edad media } \\
\text { - } \quad \text { Alta deserción escolar y analfabetismo } \\
\text { - } \quad \text { Existencia de familias numerosas } \\
\text { Baja calidad de las viviendas y deficiencia } \\
\text { en la infraestructura de servicios y } \\
\text { equipamiento }\end{array}$ & $\begin{array}{l}\text { - Disponibilidad y acceso a la } \\
\text { vivienda } \\
\text { - Utilización de áreas de menor } \\
\text { riesgo para la localización } \\
\text { residencial Facilidad de acceso a } \\
\text { los servicios sociales básicos } \\
\text { - Acceso y calidad de la educación } \\
\text { básica } \\
\text { - Cuidado de la salud y nutrición } \\
\text { - Difusión de métodos } \\
\text { anticonceptivos } \\
\text { - Creación de empleos }\end{array}$ \\
\hline $\begin{array}{l}\text { Vulnerabilidad } \\
\text { Media - Alta }\end{array}$ & $\begin{array}{ll}\text { - } & \text { Pobreza urbana } \\
\text { - } & \text { Proliferación de la marginalidad urbana } \\
\text { - } & \text { Carencia en la infraestructura de servicios } \\
\text { básicos y de equipamiento } \\
\text { - } & \text { Hacinamiento y deterioro de la viviendas } \\
\text { - } & \text { Alta fecundidad y mortalidad } \\
\text { - } & \text { Deficiencias en la seguridad social } \\
\text { - } & \text { Altos índices de dependencia } \\
\text { - } & \text { Desocupación y subocupación } \\
\text { - } & \text { Educación incompleta }\end{array}$ & 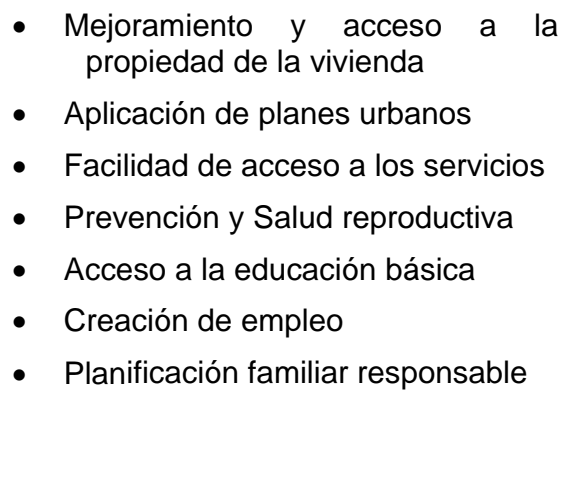 \\
\hline $\begin{array}{c}\text { Vulnerabilidad } \\
\text { Media }\end{array}$ & $\begin{array}{l}\text { - } \quad \text { Alta desocupación urbana } \\
\text { - } \quad \text { Pobres urbanos, cercanos a la línea de } \\
\text { pobreza } \\
\text { - } \quad \text { Escasa inserción de los pobres rurales } \\
\text { - } \quad \text { Educación incompleta } \\
\text { - } \quad \text { Predominio de trabajo informal } \\
\text { - } \quad \text { Tasa de Mortalidad media - alta }\end{array}$ & 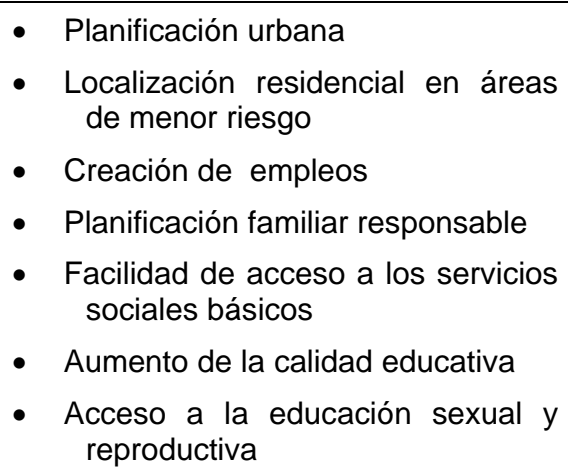 \\
\hline $\begin{array}{l}\text { Vulnerabilidad } \\
\text { Media-Baja }\end{array}$ & $\begin{array}{l}\text { - } \quad \text { Elevada Población urbana } \\
\text { - } \quad \text { Mayor incidencia de hogares con adultos } \\
\text { mayores } \\
\text { - } \quad \text { Bajos niveles de masculinidad }\end{array}$ & $\begin{array}{l}\text { - } \text { Creación de empleos } \\
\text { - Acceso y calidad de la educación } \\
\text { básica y superior } \\
\text { - Difusión de la práctica del ahorro }\end{array}$ \\
\hline
\end{tabular}




\begin{tabular}{|c|c|c|}
\hline & $\begin{array}{l}\text { Valores de Edad media más alta (23-24 } \\
\text { años) } \\
\text { - } \quad \text { Ingresos por sobre la línea de pobreza } \\
\text { - } \quad \text { Posibilidad de obtener vivienda propia } \\
\text { - } \quad \text { Trabajo asalariado del sector servicios y } \\
\text { comercio. }\end{array}$ & $\begin{array}{l}\text { - } \text { Seguridad social } \\
\text { - } \quad \text { Acceso a los créditos }\end{array}$ \\
\hline $\begin{array}{l}\text { Vulnerabilidad } \\
\text { Baja }\end{array}$ & $\begin{array}{ll}\text { - } & \text { Elevada proporción de población urbana } \\
\text { - } & \text { Baja mortalidad infantil y de la niñez } \\
\text { - } & \text { Población sin NBI } \\
\text { - } & \text { Ingresos altos } \\
\text { - } & \text { Acceso a viviendas propia con servicios } \\
\text { completos } \\
\text { - } & \text { Educación completa } \\
\text { - } & \text { Profesionales jóvenes y adultos } \\
\text { - } & \text { Población activa dedicada a actividades } \\
\text { - } & \text { Marciarias } \\
\text { - } & \text { Aumento de los riesgos asociados a la } \\
\text { vida urbana }\end{array}$ & $\begin{array}{l}\text { - } \text { Cuidado del medio ambiente } \\
\text { - } \quad \text { Atención de la tercera edad } \\
\text { - } \quad \text { Acceso a complejos asistenciales } \\
\text { de alta complejidad } \\
\text { - } \quad \text { Seguridad social } \\
\text { - Inversión social } \\
\text { - } \quad \text { Mayor propensión al ahorro } \\
\text { - } \quad \text { Formación de grupos sociales }\end{array}$ \\
\hline
\end{tabular}

\section{c. La segunda transición demográfica}

Los cambios que se produjeron en el comportamiento de la fecundidad en los países desarrollados nos introduce al tratamiento de la llamada segunda transición demográfica que implica modificaciones en los patrones de reproducción de las familias y de la nupcialidad (aumento de la edad al casarse, uniones de hecho, procreación extramatrimonial, disoluciones matrimoniales), que condujo a índices de fecundidad por debajo de los niveles de reemplazo (2,1 Hijos por mujer) y al crecimiento negativo de algunos países. (Chackiel, 2004)

La segunda transición demográfica difiere de los otros dos procesos, pues se trata de un fenómeno propio de los países desarrollados, aunque hay indicios parciales de su extensión al resto del mundo, con la generación de riesgos sociodemográficos asociados a su retraso o a su avance. Esta expresión fue difundida por demógrafos europeos (Ron Lesthaeghe y Dirk van de Kaa -1986) para describir el conjunto de cambios en las conductas matrimoniales y reproductivas de Europa en la década de 1960.

La alta fecundidad está asociada generalmente a los sectores con menores ingresos, los más pobres, de bajo nivel de instrucción y radicados en áreas rurales y urbanas marginales, entre los que se encuentran los aborígenes; mientras los grupos que han logrado bajar los índices de fecundidad son los más altos y los de mayor instrucción, por tener mayor acceso a la información necesaria para planificar los nacimientos y a los medios para controlar la natalidad. El menor número de hijos deseados por las mujeres de condición social más baja está relacionado con los cambios culturales en cuanto al ideal de hijos, a las condiciones económicas adversas para la crianza y a la incorporación de la idea de familia más pequeña, recibida a través de la influencia de diferentes medios de difusión. Al mismo tiempo la baja de la fecundidad está relacionada con el aumento del uso de métodos anticonceptivos.

El fenómeno del cambio de los comportamientos matrimoniales y reproductivos, en los países desarrollados se traduce en índices de fecundidad muy inferiores al nivel de reemplazo (postransición demográfica clásica), el incremento de la soltería, los nacimientos fuera del matrimonio, la postergación de las uniones y de la maternidad y la paternidad hasta el momento de haber alcanzado un mínimo nivel de formación educativa y de obtener una posición socioeconómica favorable para encarar las distintas etapas y acontecimientos de la vida. Es por ello que la población joven de esos países contraen matrimonio y tiene su primer hijo a edades significativamente mayores que sus pares de los países subdesarrollados. 
Esta manifestación contempla también un aumento de las uniones ilegítimas, de los divorcios, separaciones, que implican dificultades en la crianza de los hijos, el abandono de la mujer propio de esos acontecimientos traumáticos, caracterizados generalmente por cambios socioeconómicos y de género, que afectan a hogares con menor capacidad de respuesta o mayor vulnerabilidad frente a esas adversidades. Cualquiera sea el ámbito que se trate, la inestabilidad familiar genera problemas para los involucrados que requieren aumentar su capacidad de respuesta frente a la posibilidad de ocurrencia. Tales cambios obedecen a una transformación cultural profunda, en la que la individualidad toma una posición central fundamentada en los valores postmodernos, en la conciencia materialista y en el logro de una mejor calidad de vida.

Las conductas reproductivas de los jóvenes surgen como elementos de vulnerabilidad, pues socialmente su comportamiento induce a un retraso de la nupcialidad, de la reproducción y de la movilidad social, pero culturalmente no se cuentan con los elementos necesarios para consolidarlos (educación, oportunidades de movilidad social y respeto a las normas) (Rodríguez Vignoli, 2001)

La educación es la principal herramienta de las sociedades modernas para promover y realzar el capital humano logrando la integración de los jóvenes y adolescentes. La opción de movilidad social depende de la educación recibida y si esta es deficiente opera como un elemento de vulnerabilidad que debilita a los grupos humanos frente a las exigentes condiciones del mundo actual. Pero no solamente se trata de desigualdades en la calidad, sino también en la cobertura de la formación educativa y en la complementación de los diferentes niveles. Es destacable el problema de la deserción escolar desde los niveles inferiores (educación básica) hasta los superiores, siendo más acentuado entre los más pobres como resultado de su incorporación temprana a los roles adultos, en particular los vinculados al sostenimiento familiar.

Actualmente, los jóvenes de estratos sociales bajos, tanto urbanos como rurales, están sometidos a procesos de vulnerabilidad debido a varios factores, entre otros:

a. la falta de oferta laboral para personas con escasa instrucción que les garantice la cobertura de los servicios sociales y la estabilidad.

b. La carencia de ofertas educativas de capacitación para adquirir nuevas destrezas o aptitudes.

c. La falta de recursos familiares para contener a los jóvenes y proporcionarles alimentación, educación y formación.

d. La estimulación social de la sexualidad temprana que no se condice con la educación y la capacitación para obtener comportamientos reproductivos y de prevención de enfermedades.

e. Aislamiento de los jóvenes urbanos pobres debido a la segregación residencial y a la restricción de los servicios básicos.

En numerosas investigaciones llevadas a cabo sobre la vulnerabilidad sociodemográfica se utilizan enfoques centrados en la identificación de las desventajas sociales que ejercen esos efectos adversos sobre los hogares y las personas. La relación de las variables de población con los daños ambientales, la salud reproductiva, la localización de áreas marginales, los activos, los ingresos, permiten examinar riesgos como la alta y temprana fecundidad, los elevados valores de dependencia demográfica, el aislamiento y la dispersión de la población. Así los riesgos de la segunda transición permite tratar los siguientes ejes temáticos: 1 . El ciclo de la vida de las personas que identifica a tramos de edades con mayor o menor vulnerabilidad (infancia, adolescencia, etapa reproductiva y laboral, adultez, vejez); 2. Los derechos de cada una de estas fases y 3. El estadio de transición demográfica que se relaciona con la segunda transición. Esto permite proponer cuatro indicadores de vulnerabilidad: a) las condiciones socioeconómicas relacionados con la salud de los niños, la educación de las madres, la calidad de las viviendas y los servicios básicos; b) la deserción escolar; c) el trabajo desprotegido y d) la falta de seguridad social. (CEPAL, 2002)

\section{d. La transición epidemiológica}

Los adelantos en la medicina, la mayor cobertura de los servicios de salud y la mejora en las condiciones de vida de la población, produjeron bajas considerables en los niveles de mortalidad. En A. Latina esos cambios se inician en la primera mitad del siglo XX. Según datos proporcionados por Naciones Unidas en los últimos 50 años, se habría ganado 20 años en promedio la esperanza de vida, aunque existen diferencias importantes entre los países. En todos ellos la mortalidad femenina es inferior a la masculina asociada a la incidencia diferencial por sexo de las enfermedades o circunstancias que causan las muertes (enfermedades cardiovasculares, violencia). Así la diferencia 
de esperanza de vida es de 6,5 años a favor de la mujer, aunque se espera que, la mayor participación de la misma en actividades fuera del hogar (que las expone a riesgos similares al de los hombres), como así el combate de enfermedades crónicas masculinas, reduzcan las diferencias. La ganancia de años en la esperanza de vida está relacionada principalmente con el descenso de la mortalidad infantil y de la niñez a raíz de la menor incidencia de enfermedades infecciosas, parasitarias y del aparato respiratorio. La elevada mortalidad infantil se registra en las poblaciones cuyas madres tienen escasa o no tienen instrucción. (Chackiel, 2004).

En el campo de la salud y en estrecha relación con la transición demográfica se expone y desarrolla la denominada teoría de la transición epidemiológica. Esta expresa el cambio de las características de la morbilidad, las causas de muertes y la distribución por edad de las defunciones. El proceso consiste en la disminución proporcional de las muertes ocasionadas por enfermedades perinatales y las transmisibles (infecciosas, parasitarias y respiratorias) dando paso a las crónicas y degenerativas (tumores, circulatorias) y las provocadas por causas externas (violencia, accidentes, traumatismos).

Si bien se viven modificaciones profundas en el cambio de las causas de muerte, existen rebrotes de enfermedades que aparentemente estaban superadas, como es el caso de la década de los 90 con el surgimiento de epidemias de enfermedades transmisibles como el cólera, la malaria, el mal de chagas, el hantavirus, la tuberculosis y el dengue, tanto en áreas rurales como urbanas, ocasionadas por la permanencia de la pobreza, la falta de servicios básicos y el alto grado de hacinamiento.

Para estudiar el cambio de los patrones de salud y enfermedad Abdel Omram (1971) formuló la teoría de la transición epidemiológica y, aunque su tratamiento data desde sus estudios, la terminología comienza a usarse en los diccionarios médicos hacia 1995. La premisa en esta teoría es que la mortalidad se constituye en un factor fundamental de la dinámica de la población y postula la transición de un patrón de causas de muerte por enfermedades infecciosas, con muy alta mortalidad, especialmente en edades jóvenes, a otro patrón dominado por las enfermedades degenerativas y las provocadas por la acción del hombre. Además se asegura que los cambios en salud y los patrones de enfermedad están estrechamente vinculados con la transición demográfica y el nivel socioeconómico de las sociedades. (Vera Bolaños, 2000)

En la dicha teoría, Omram plantea que el desarrollo histórico de la mortalidad se caracteriza por transcurrir en tres etapas diferentes: la edad de la peste y el hambre, la edad de las pandemias retraídas y la edad de las enfermedades degenerativas y las causadas por el hombre.

a. en la edad de la peste y el hambre la mortalidad fue alta y fluctuante, lo que provocó un crecimiento escaso de la población. Además la esperanza de vida era baja (entre 20 y 40 años).

b. En la edad de las pandemias retraídas las crisis de la mortalidad se vuelven menos frecuentes y la esperanza de vida se incrementó (entre 30 y 50 años). Por otra parte el crecimiento demográfico fue sostenido.

c. En la edad de las enfermedades degenerativas y las producidas por el hombre, la mortalidad es baja y estable; la esperanza de vida aumenta hasta alcanzar a más de 50 años. La fecundidad se convierte en el hecho decisivo para controlar el crecimiento de la población.

Las condiciones de salud inciden directamente sobre la productividad (ausentismo laboral, envejecimiento prematuro, disminución de la vida activa) al mismo tiempo que influyen en las condiciones económicas (deterioro social e incremento de la pobreza). Un problema grave es el trabajo de los menores de 14 años, sector expuesto a factores de riesgo que derivan en cuadros patológicos que afectan las potencialidades futuras de la población activa. (Rivadeneira, 2000)

La mejora de las condiciones de salud de las mujeres y de los niños contribuirá a disminuir la mortalidad materna e infantil y a reducir indirectamente los niveles de fecundidad (especialmente embarazo adolescente). De esa forma, la salud y la educación son factores gravitantes para lograr el mejoramiento de los recursos humanos, tendiente a elevar los niveles de productividad. Así por ejemplo, la tasa de mortalidad materna en Argentina paso de 56 muertes de madres cada mil nacimientos en 1988 a 35 en el año 2000. América Latina tenía en promedio en 1995, 190 muertes c/1000 nacidos. (Chackiel, 2004) 
En la transición epidemiológica (algunas veces referida como transición de la mortalidad o transición en salud) estrechamente vinculada a la transición demográfica y de acuerdo con la situación demográfica y de salud de los países se pueden distinguir 4 fases: (www.webmastersanitarios.org.)

Primera fase: comprende la epidemiología de los países más pobres, con un predominio de enfermedades infecciosas y de la nutrición, con una mortalidad infantil muy alta, mortalidad general por causas cardiovasculares baja y esperanza de vida de la población relativamente corta. Los procesos degenerativos son poco frecuentes por las características de la alimentación.

Segunda fase: Corresponde a los países en vías de desarrollo donde el mejor nivel socioeconómico se corresponde con una mejora en la alimentación y en las condiciones higiénicas y sanitarias, con lo cual disminuyen las enfermedades infecciosas (aunque con una importante prevalencia) y de la nutrición. Además aparecen en forma incipiente los factores de riesgo cardiovasculares y aumenta la incidencia de la arteriosclerosis.

Tercera fase: Incluye a los países en transición, donde el importante desarrollo económico acompaña a los cambios nutricionales (alimentación rica en grasas, sal) y al estilo de vida (vida sedentaria, aumento del estrés, la obesidad y el consumo de tabaco y alcohol). Ello conduce a la modificación de la distribución de las enfermedades, con mayores factores de riesgo cardiovasculares y accidentes cerebro vasculares. Existe una mayor esperanza de vida y una disminución importante de las enfermedades infecciosas y de la nutrición.

Cuarta fase: Comprende a los países más desarrollados con mayores avances en la investigación médica y con la aplicación de medidas de educación sanitaria que ayudan a tomar conciencia a la población sobre el riesgo de las enfermedades cardiovasculares especialmente. De esa manera se disminuye el consumo de alimentos nocivos, se incentiva la práctica de los ejercicios físicos, se evita la obesidad, el estrés y el consumo de tabaco. Todo eso conlleva a la reducción de la morbilidad y mortalidad, como asimismo a la prolongación de la esperanza de vida. Sin embargo todavía existen diferencias entre las capas altas y bajas de esas sociedades.

La transición epidemiológica no siempre se presenta estrictamente en esas cuatro fases, sino que existen otros determinantes, distintos del nivel socioeconómico, que pueden alterar la incidencia de las enfermedades sobre la población y, de esa forma, la vulnerabilidad a contraer ciertas enfermedades que parecieran superadas.

Durante la misma los cambios más profundos en salud y enfermedades ocurren entre los niños y las mujeres, por ser los grupos más vulnerables a esos procesos. Al mismo tiempo esos cambios están estrechamente vinculados con otros procesos transicionales que constituyen la compleja modernización.

A propósito de las particularidades del cambio y su variación temporal, se distinguen tres modelos básicos de transición epidemiológica (Vera Bolaños, 2000):

1. El modelo oeste o clásico que describe un cambio gradual de tasas de mortalidad altas (30 por mil) y natalidad alta (40 a 50 por mil) a tasa bajas 10 y 20 por mil respectivamente, como consecuencia de las mejoras sociales, ambientales y económicas y a la práctica de métodos de control de la natalidad, donde poco tiene que ver los adelantos en la medicina, por lo menos en las etapas iniciales.

2. El modelo acelerado tiene un comportamiento similar al anterior pero fue mucho más brusco el cambio debido a la trascendencia de la tecnología médica.

3. El modelo tardío presenta una mortalidad en descenso por efecto fundamental de la revolución médica en gran parte importada. La fecundidad tuvo un descenso tardío por efecto de los métodos de control.

\section{Reflexiones finales}

La noción de vulnerabilidad social y demográfica ayuda a comprender e identificar a los grupos humanos, hogares e individuos que por sus características comunes quedan expuestos a sufrir cambios significativos. Al mismo tiempo se relaciona con la línea conceptual de la marginación y la pobreza y con las distintas condiciones de riesgo. El término no se agota en ellos, sino más bien los incluye. 
Evidentemente los procesos de cambio de comportamiento de los hechos humanos y sociales, como así también, las transformaciones ocasionadas en las distintas transiciones, vinculadas con la vulnerabilidad sociodemográfica, afectan a las poblaciones de manera diferente y de acuerdo con sus características sociales, económicas, demográficas y ambientales. Es por ello que, el análisis del comportamiento diferencial de los grupos humanos frente a los riesgos, de acuerdo al grado de vulnerabilidad global de los mismos, debe contemplar la capacidad de respuesta de ellos.

Los riesgos sociodemográficos no se pierden, sino que se modifican. El avance de las distintas transiciones reduce algunos, pero no impide que otros persistan y algunos nuevos aparezcan, es decir la inestabilidad que contiene la dinámica demográfica no termina con la supuesta estabilización de la población. El análisis y la evaluación de las vulnerabilidades, realizados desde distintas ópticas permite detectar la susceptibilidad a los riesgos, diagnosticar la calidad de vida de la población en áreas espaciales localizadas y en sectores marginados de la población, comparar el estado del equipamiento de los servicios, analizar los factores socioculturales, indagar sobre aspectos de la percepción de la población sobre los riesgos, analizar pautas de comportamiento económico en relación con el deterioro de la capacidad productiva, evaluar actitudes y prácticas de la población, entre otros. El conocimiento del comportamiento diferencial de las distintas variables que afectan a los grupos humanos y a la vulnerabilidad de los mismos frente a los hechos, permitirá esbozar estrategias sociales para conocer y mejorar las actitudes y prácticas de la población, como así formular recomendaciones, en base de la experiencia acumulada, para implementar políticas ambientales y sociales que mitiguen los riesgos.

\section{REFERENCIAS BIBLIOGRÁFICAS}

- Arriagada Luco, Camilo (2003) La dinámica demográfica y el sector habitacional en América Latina. Serie Población y Desarrollo, N³3, Santiago de Chile, CEPAL.

- Busso, Gustavo (2002) Vulnerabilidad. Socio-demográfica en Nicaragua: un desafío para el crecimiento económico y la reducción de la pobreza. Serie Población y Desarrollo, N²9, Centro Latinoamericano y Caribeño de Demografía (CELADE), Santiago de Chile.

- CEPAL (2002) Vulnerabilidad sociodemográfica: viejos y nuevos riesgos para comunidades, hogares y personas. Síntesis y conclusiones. Brasilia (versión electrónica)

- Chackiel, Juan (2004) La dinámica demográfica en América Latina. Serie Población y Desarrollo, N052, Santiago de Chile, CEPAL

- Chackiel, Juan y Schkolnik, Susana (2003) América Latina: los sectores rezagados en la transición de la fecundidad. Serie Población y Desarrollo, N42, Centro Latinoamericano y Caribeño de Demografía (CELADE), Santiago de Chile.

- Pinto da Cunha, José Marcos (2002) Urbanización, redistribución espacial de la población y transformaciones socioeconómicas en América Latina. Serie Población y Desarrollo N³0, Santiago de Chile, CELADE.

- Rivadeneira S, Luis (2000) América Latina y el Caribe: crecimiento económico sostenido, población y desarrollo. Serie Población y Desarrollo №2, Santiago de Chile, CELADE.

- Rodríguez Vignoli, Jorge (2001) Vulnerabilidad y grupos vulnerables: un marco de referencia conceptual mirando a los jóvenes. Serie Población y Desarrollo, No17, Santiago de Chile, CEPAL.

- Rodríguez Vignoli, Jorge (2002) Distribución territorial de la población de América Latina: tendencias, interpretaciones y desafíos para las políticas públicas. Serie Población y Desarrollo No32, Santiago de Chile, CELADE.

- Vera Bolaños, Marta (2000) Revisión crítica a la Teoría de la transición epidemiológica. Papeles de población N²5, CIEAP/UAEM, México.

- www.webmastersanitarios.org. La transición epidemiológica. 2004. 